\title{
Psychoserisikostadien als eigene Diagnosekategorie - Pro
}

\author{
Risk States for Psychosis as a Distinct Diagnostic Category - Pro
}

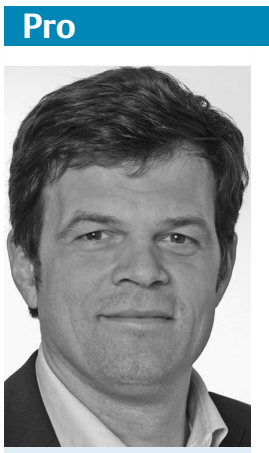

Andreas Bechdolf
In Anbetracht der begrenzten Effektivität der vorhandenen Behandlungsoptionen hat der Direktor des National Institut of Health in den USA, T. R. Insel, stellvertretend für viele Wissenschaftler, Prävention als die aussichtsreichste Strategie eingeschätzt, den Krankheitsverlauf der schizophrenen Störung und seiner Folgen für die Betroffenen, Angehörigen und die Gesellschaft positiv zu beeinflussen [1].

Mit dem „abgeschwächten Psychosesyndrom“, wie es jetzt in die Forschungskriterien von DSM-5 aufgenommen wurde, ist ein wichtiger Schritt von der Therapie hin zur Prävention schizophrener Störungen vollzogen worden [2]. Hiermit wurde ein wesentliches Teilsyndrom der Risikokriterien für psychotische Ersterkrankungen, die in den letzten 20 Jahren entwickelt und evaluiert wurden, in die diagnostischen Kriterien aufgenommen. Obwohl das Übergangsrisiko in die erste psychotische Episode und die darauf bezogene Prävention den Hauptfokus dieser Forschungsrichtung bildet, wurde mit zunehmendem Kenntnisstand deutlich, dass das Risikosyndrom für sich genommen, unabhängig vom Übergang in die Psychose, Krankheitswert hat [3]. Aus diesem Grund, um die Betroffenen weniger $\mathrm{zu}$ stigmatisieren und um die Offenheit des Ausgangs eines Risikosyndroms zu einer nicht psychotischen Erkrankung oder in die Remission zu betonen, wurde in der finalen Version des DSM-5 von einem Begriff, der den Ausgang des Syndroms in den Mittelpunkt stellt (Psychoserisikostadium) abgesehen und für ein rein deskriptiven, querschnittlichen Begriff entschieden (abgeschwächtes Psychosesyndrom) [2].

Generell ist zu betonen, dass es sich bei der Prävention von Psychosen, wie sie derzeit betrieben wird, nicht um den Versuch einer Primärprävention universal in der Allgemeinbevölkerung oder selektiv bei noch gesunden Risikoträgern handelt. Dazu ist die Inzidenz der schizophrenen Störung, wie von R. Warner völlig korrekt dargestellt, zu niedrig. Stattdessen handelt es sich derzeit um eine indizierte Prävention bei Personen, die bereits klinische Symptome zeigen und wegen der damit verbundenen Beschwerden und Einschränkungen aktiv Hilfe suchen. In dieser Population ist die Inzidenz der schizophrenen Störung wesentlich höher und Screenings bezüglich des abgeschwächten Psychosesyndroms können sinnvoll eingesetzt werden.

Die Psychoserisikokriterien wurden in prospektiven Studien bei hilfesuchenden, klinisch symptomatischen Jugendlichen und jungen Erwachsene rund um die Welt evaluiert. In einer aktuellen Metaanalyse, welche die Daten von etwa 2500 Betroffenen einbezog, wurden Übergangsraten in die psychotische Ersterkrankung von $18 \%$ nach 6 Monaten, $22 \%$ nach 1 Jahr und 29\% nach 2 Jahren und $36 \%$ nach 3 Jahren festgestellt [8]. Die Betroffenen zeigen also ein klinisch bedeutsames Risiko einer psychotischen Erstmanifestation, welches mehrere 100-fach über dem Erkrankungsrisiko der Normalbevölkerung liegt.

Zur indizierten Prävention bei Personen mit erhöhtem Psychoserisiko liegen derzeit 8 abgeschlossene randomisiert-kontrollierte Studien mit etwa 1000 Teilnehmern vor, welche low-dose Risperidon und kognitive Verhaltenstherapie, kognitiv verhaltenstherapeutische Einzel- oder Komplexinterventionen, Olanzapin oder Omega-3-Fettsäuren bei Patienten mit abgeschwächten psychotischen Symptomen evaluiert haben. Mittlerweile zeigen 3 Metaanalysen [5 -7], dass die jeweiligen Experimentalbedingungen das Auftreten erster psychotischer Episoden in klinisch bedeutsamen Ausmaß mindestens über 1 - 2 Jahre verzögern können, die Symptomatik bessern und überwiegend auch das
Funktionsniveau. Derzeit empfehlen die meisten Autoren, wegen des günstigeren Nutzen/Risiko-Profils zunächst psychosoziale Interventionen anzubieten und nur bei besonderen Risiken oder Nonresponse eine niedrig dosierte antipsychotische Medikation vorzuhalten [5-8]. Leider ist die Vermutung von R. Warner zutreffend, dass viele Patienten mit abgeschwächtem Psychosesyndrom ungerechtfertigterweise medikamentös antipsychotisch behandelt werden. In einer eigenen Studie war das bei 139 von 503 Betroffenen der Fall [9]. Dass diese Rate durch die Einführung des Syndroms in DSM-5 erhöht wird, wie von R. Warner formuliert, ist erstmal eine Hypothese. Das Gegenteil halte ich für wahrscheinlicher: Die hohe Antipsychotikaverschreibung in dieser Patientengruppe, könnte gerade dadurch begründet sein, dass bis dato keine Diagnose für dieses Syndrom zu Verfügung steht und entsprechend auch keine Leitlinienempfehlungen vorliegen, die psychotherapeutische Behandlung empfehlen. R. Warners Vermutung, Betroffene würden durch die Identifikation des Syndroms und dessen Behandlung stigmatisiert, ist weit verbreitet, aber nach ersten Pilotergebnissen nicht zutreffend. Betroffene beschreiben Aufklärung und psychoedukativ-psychotherapeutische Angebote als hilfreich und entlastend [10].

Personen mit erhöhtem Psychoserisiko leiden unabhängig vom Übergang in die Psychose unter massiv eingeschränkter Lebensqualität, sowie reduziertem Funktionsniveau. 60-80\% der Betroffenen erfüllen zusätzlich zum abgeschwächten Psychosesyndrom andere Diagnosekriterien, am häufigsten Depression, Angststörungen und Substanzmissbrauch. Viele Betroffene haben oft eine lange frustrierende Suche nach Unterstützung hinter sich. Besonders bedeutsam ist, dass auch Personen, die nicht in die Psychose übergehen im 2-Jahres-Verlauf zu fast 50\% weiterhin abgeschwächte Psychosesymptome zeigen und insgesamt gegenüber einer Vergleichsgruppe ein deutlich redu- 
ziertes Funktionsniveau aufweisen (Übersicht bei [5]). Die Behauptung von R. Warner, dass die Symptome häufig spontan remittieren ist somit deutlich zu relativieren. Der überwiegende Teil der Betroffenen ist lang andauernd symptomatisch und funktionseingeschränkt oder geht in eine Psychose über.

Obwohl zweifelsohne noch weitere Forschungsbemühungen in dem Feld notwendig sind, ist die Einführung des Syndroms in die Regelversorgung vor dem Hintergrund der dargestellten Daten als gerechtfertigt anzusehen. Um die Kostenträger von dem dringend notwendigen Aufbau eines spezifischen Versorgungsangebots für Personen mit abgeschwächtem Psychosesyndrom zu überzeugen, ist mindestens in der BRD eine spezifische ICDCodierung des Syndroms Voraussetzung. In Anlehnung an DSM-5 ist das für ICD11 geplant [11].

Ein spezifisches Versorgungsangebot sollte niederschwellig, nicht stigmatisierend und überwiegend ambulant konzipiert sein sowie die spezifischen Bedürfnisse von jungen Erwachsenen und Jugendlichen reflektieren. Innerhalb dieser Zentren sollen eine Reihe von Interventionsmöglichkeiten vorgehalten werden. Diese reichen von einem regelmäßigen Monitoringtermin, motivierender Gesprächsführung bei Substanzmissbrauch, supportiver Therapie und kognitiver Therapie bis hin zur pharmakologischen Intervention und aufsuchenden Behandlung (Details s. [12]).
Ein solches Versorgungsmodell ist keine Fiktion. In Australien und Großbritannien sind groß angelegte Gesundheitsreformen im Gange, bei denen jugendfreundliche Interventionszentren gegründet werden, die außerhalb von klinischen Settings in Beratungs- und Jugendzentren etabliert werden, um Patienten mit abgeschwächten Positivsymptomen und ersten Episoden psychiatrischer Störungen gut zu erreichen und frühzeitig $\mathrm{zu}$ behandeln $[13,14]$.

\section{Literatur}

1 Insel TR. Rethinking schizophrenia. Nature 2010; 468: 187-193

2 Association AP. Diagnostic and Statistical Manual of Mental Disorders: Dsm-5. Amer Psychiatric Pub Incorporated; 2013

3 Tsuang MT, Van Os J, Tandon R et al. Attenuated psychosis syndrome in DSM-5. Schizophr Res 2013; 150: 31 - 35

4 Fusar-Poli P, Bonoldi I, Yung AR et al. Predicting psychosis: meta-analysis of transition outcomes in individuals at high clinical risk. Arch Gen Psychiatry 2012; 69: 220 229

5 Fusar-Poli P, Borgwardt S, Bechdolf A et al. The Psychosis High-Risk State - A Comprehensive State-of-the-Art Review. JAMA psychiatry 2013; 70: 107-120

6 Preti A, Cella M. Randomized-controlled trials in people at ultra high risk of psychosis: a review of treatment effectiveness. Schizophr Res 2010; 123: 30-36

7 van der Gaag M, Smit F, Bechdolf A et al. Preventing a first episode of psychosis: Metaanalysis of randomized controlled prevention trials of 12 month and longer-term follow-ups. Schizophr Res 2013; 149: 56-62

8 Müller H, Wießmann T, Bechdolf A. Interventionen bei Personen mit erhöhtem Psychoserisiko: Eine aktuelle Übersicht über randomisiert kontrollierte Studien. Fortschr Neurol Psychiatr 2012; 80: 570-579
9 Bechdolf A, Mueller H, Stuetzer H et al. Rationale and baseline characteristics of PREVENT: a second-generation intervention trial in subjects at-risk (prodromal) of developing first-episode psychosis evaluating cognitive behavior therapy, aripiprazole, and placebo for the prevention of psychosis. Schizophr Bull 2011; 37 (Suppl. 02): S111 121

10 Hauser M, Lautenschlager M, Gudlowski Y et al. Psychoeducation with patients at-risk for schizophrenia - an exploratory pilot study. Patient Educ Couns 2009; 76: 138-142

11 Gaebel W. Status of psychotic disorders in ICD-11. Schizophr Bull 2012; 38: 895-898

12 Häfner $H$, Bechdolf A, Klosterkötter $J$ et al. Psychosen - Früherkennung und Frühintervention: Der Praxisleitfaden. Stuttgart: Schattauer; 2012

13 Australia Co. A Ten Year Roadmap for National Mental Health. 2012

14 www.youthspace.me

Sie haben eine eigene Meinung zu diesem Thema? Dann schreiben Sie uns an: psychiat-praxis@thieme.de!

\section{Korrespondenzadresse}

\section{Prof. Dr. med. Andreas Bechdolf, M. Sc.}

Kliniken für Psychiatrie, Psychotherapie und Psychosomatik, Vivantes Klinikum am Urban, Vivantes Klinikum im Friedrichshain

Dieffenbachstraße 1

10967 Berlin

andreas.bechdolf@vivantes.de

Bibliografie

Dol http://dx.doi.org/

10.1055/s-0034-1369836

Psychiat Prax 2014; 41: 124-125

(c) Georg Thieme Verlag KG

Stuttgart · New York

ISSN 0303-4259 